\title{
Efficacy of Bronchoscopic Closure of a Bronchopleural Fistula with Amplatzer Devices: Long-Term Follow-Up
}

\author{
Oren Fruchter ${ }^{a, b} \quad$ Bayya Abed El Raouf ${ }^{b} \quad$ Nader Abdel-Rahman $^{b} \quad$ Milton Saute $^{c}$ \\ Elchanan Bruckheimer ${ }^{a, d}$ Mordechai R. Kramer ${ }^{a, b}$ \\ a Sackler School of Medicine, Tel Aviv University, Tel Aviv, and b Pulmonary Institute and ' Department of \\ Cardiothoracic Surgery, Rabin Medical Center, and dDepartment of Pediatric Cardiology, Schneider Children's \\ Medical Center of Israel, Petah Tiqva, Israel
}

\section{Key Words}

Bronchopleural fistula · Bronchoscopy · Vascular

embolization coil · Amplatzer occlusion · Thoracic surgery

\begin{abstract}
Background: The development of a bronchopleural fistula (BPF) is associated with high rates of morbidity and mortality. We have developed a minimally invasive method of bronchoscopic BPF closure using Amplatzer devices (AD) and Amplatzer vascular plugs (AVP), with excellent shortterm results. Objectives: The aim of the present report was to explore the long-term outcome of patients treated by Amplatzer occluders and the durability of this novel modality of BPF treatment. Methods: A total of 31 central BPF in 31 patients (mean age 66.8 years, range 19-91) were sealed under moderate sedation bronchoscopically by either $A D(n=19)$ or AVP $(n=12)$. The average follow-up period was 17.6 months (range 1-68 months). Results: The main etiology for BPF was surgery $(n=24)$, pneumonectomy $(n=14)$ or lobectomy/segmentectomy $(n=10)$. The underlying disease was either primary $(n=19)$ or metastatic $(n=2)$ lung cancer. The immediate success rate was $96 \%$ as symptoms related to BPF disappeared in 30 of the 31 patients. Short-term ( $<30$ days) mortality was $13 \%$ (4 patients). At follow-up, 14 patients $(45 \%)$ are still alive. Out of 12 patients with late mortality, in
\end{abstract}

5 patients (41\%) the death was directly related to cancer relapse, and no patient died due to BPF recurrence. Conclusion: Endobronchial closure of BPF using both types of Amplatzer occluders ( $A D$ and AVP) is a minimally invasive effective modality of treatment with high safety profile and satisfactory long-term outcome considering the poor prognosis in this particular group of patients.

(c) 2014 S. Karger AG, Basel

\section{Introduction}

Central bronchopulmonary fistulas (BPFs) are associated with high rates of morbidity and mortality $[1,2]$. They occur as an uncommon, but often severe, complication of surgical procedures involving pulmonary resection for cancer, in particular pneumonectomy, with an estimated incidence ranging between 3 and $28 \%[3,4]$. The incidence of BPF following lobectomy is significantly lower $(0.5 \%)$ [3]. The mortality rate associated with BPF ranges from 16 to $72 \%$ [1-4].

Oren Fruchter and Bayya Abed El Raouf contributed equally to the study.

\section{KARGER}

E-Mail karger@karger.com

www.karger.com/res (c) 2014 S. Karger AG, Basel

0025-7931/14/0873-0227\$39.50/0
Prof. Mordechai R. Kramer, MD, FCCP

Pulmonary Institute

Rabin Medical Center, Beilinson Campus

Petah Tiqva, IL-49100 Tel Aviv (Israel)

E-Mail kremerm@clalit.org.il 
BPFs are significantly more prevalent in patients with malignancy, as opposed to benign conditions. Additional risk factors for their development include external radiation and chemotherapy [4].

Treatment of central BPF includes a range of surgical and medical techniques: chest drains, Eloesser muscle flap, omental flap, transsternal bronchial closure, thoracoplasty and prolonged antibiotic regimens [3]. Endoscopic treatment of BPF is based on the delivery of biological glue, coils, covered stents and sealants to the BPF site [5-14]. The degree of success with bronchoscopic treatment is variable, and depends on the underlying disease, and the proximity and size of the fistulas, with larger fistulas having poor closure rates [6-9]. In previous reports $[15,16]$, our group described a novel method to close large BPF in 12 patients using the Amplatzer septal and ductal occlusion that is traditionally used for transcatheter closure of atrial septal defects and patent ductus arteriosum. This novel treatment is suitable only for patients with large BPF and long tract communicating between the bronchial system and the pleural cavity. For patients with small BPF that are sinus shaped, we described [17] a method of bronchoscopic closure using Amplatzer vascular plugs (AVP) that can be introduced through the working channel of the bronchoscope and deployed under direct vision to seal small BPFs. The major limitation of our previous reports is the relatively short duration of the postprocedural follow-up. Consequently, in the current study, we describe the long-term outcome of patients with BPF successfully occluded by either Amplatzer device (AD) or AVP.

Early results of the first $10 \mathrm{AD}$-treated patients and the first 5 AVP-treated patients treated have been described by us previously $[16,17]$.

\section{Patients and Methods}

A permission to collect data from Rabin Medical Center records of patients that underwent AD placement between March 2007 and April 2013 was obtained from the Institutional Review Board of the Rabin Medical Center (approval No. 0128-12).

Written informed consent was obtained from all patients before the procedure, and all patients were informed on the fact that an off-label use of the device is being used.

All the procedures were carried out at the bronchoscopy suite under moderate sedation using a combination of midazolam (1-5 mg) and alfentanil (0.5-1 mg).

A fiberoptic bronchoscope (BF-1T240 Video Bronchoscope; Olympus Corporation, Tokyo, Japan, or Pentax FB-18X Fiber Bronchoscope, Pentax, Tokyo, Japan) was introduced transnasally and advanced to view the BPF.
Following direct visualization of the fistula, $1 \mathrm{ml}$ of contrast medium (Ultravist; Bayer, Leverkusen, Germany) was diluted with an equal volume of saline solution, and $10 \mathrm{ml}$ of the mixture were drawn into a 10-ml syringe and injected under cineangiographic guidance. A dynamic bronchogram was recorded, delineating the BPF length and its shape.

Different characteristics of the fistula, such as location, depth and shape, as well as anatomical characteristics of the tracheobronchial tree, were analyzed and used to guide the decision to use either AD or AVP for closing the BPF. In cases in which the BPF had a long $(>10 \mathrm{~mm}$ ) neck, $\mathrm{AD}$ was usually used, whereas small short-necked and pin-shaped BPFs were sealed using AVP.

The methods for bronchoscopic deployment of AD and AVP have been described by our group in detail previously [15-17], hence only a short description of both techniques is provided.

\section{Amplatzer Devices}

Amplatzer double-disk occluders (AGA Medical, Golden Valley, Minn., USA) represent a large family of devices designed originally for transcatheter closure of cardiac septal defects or patent ductus arteriosus. The devices are made of a nitinol mesh with a central connector between the disks. In this series, we used the Amplatzer atrial septal occluder (ASO) and Amplatzer ductal occluder II (ADOII) [18-20]. ASOs have a central waist between the disks ranging from 4 to $40 \mathrm{~mm}$ in diameter, and the distal and proximal disk diameters are 14 and $10 \mathrm{~mm}$ larger, respectively, than the diameter of the central waist, in order to provide an anchoring lip of 5-7 mm circumferentially. A polyester fabric inside the mesh facilitates occlusion and tissue growth over the device. The waist is placed inside the defect, and the disks anchor the device on either side. ADOII behaves in a similar manner but has a central waist 3-6 $\mathrm{mm}$ in diameter, and varies in length from 4 to $6 \mathrm{~mm}$. The disks are $6 \mathrm{~mm}$ larger than the central waist. Owing to the superelastic and shape memory properties of nitinol, the devices can be collapsed into low-profile delivery sheaths (5-7 F) for advancement across the lesion.

\section{Technique of Deployment}

Amplatzer Device. Under direct bronchoscopic and fluoroscopic visualization, a 0.035 -inch guide wire was introduced through the fistula $[15,16]$. Bronchography was performed to outline the anatomy of the fistula, in particular its length. Over the wire a 5- to 7-french delivery sheath was introduced through the defect. The size and type of AD chosen was dependent on the diameter of the bronchial stump and the length of the fistula with the intent that the retention disk of the occluder would completely cover the fistulous connection and fit inside the stump. The AD was delivered by extruding a disk distal to the defect and then pulling the sheath back to oppose the disk to the defect. The central waist was then extruded in the defect, and the second disk was placed on the proximal side of the defect. Once positioned correctly, the device was released by unscrewing the attached cable. A dynamic bronchogram was performed by injecting contrast medium through the delivery sheath to check device position in relation to the stump and fistula, and bronchoscopy demonstrated the disk to completely cover the fistula and if these were satisfactory, the device was detached.

Amplatzer Vascular Plug. The AVP (AGA Medical, Golden Valley, Minn., USA) is a self-expandable cylindrical occluding de- 
Table 1. Clinical and demographic characteristics of $19 \mathrm{BPF}$ patients treated with AD placement

\begin{tabular}{|c|c|c|c|c|c|c|c|c|}
\hline & Gender & $\begin{array}{l}\text { Age, } \\
\text { years }\end{array}$ & Main diagnosis & Surgical treatment & $\mathrm{AD}, \mathrm{mm}$ & $\begin{array}{l}\text { Survival, } \\
\text { months }\end{array}$ & Death & Cause of death \\
\hline 1 & male & 69 & lung SQ cell cancer & RML lobectomy & $6 \times 6$ & 5.9 & no & \\
\hline 2 & male & 62 & lung adenocarcinoma & right pneumonectomy & $6 \times 4$ & 39.2 & no & \\
\hline 3 & male & 67 & lung adenocarcinoma & LUL + LLL lobectomy & $6 \times 4$ & 41.4 & no & \\
\hline 5 & male & 78 & lung adenocarcinoma & right pneumonectomy & $6 \times 6$ & 42.1 & no & \\
\hline 6 & male & 68 & lung adenocarcinoma & right lobectomy & $6 \times 6$ & 37.2 & no & \\
\hline 7 & female & 42 & lung SQ cell cancer & right pneumonectomy & $6 \times 6$ & 10.5 & no & \\
\hline 8 & male & 62 & lung adenocarcinoma & left pneumonectomy & $6 \times 6$ & 8.4 & yes & disease recurrence \\
\hline 11 & male & 67 & lung SQ cell cancer & right pneumonectomy & $6 \times 6$ & 2.2 & yes & disease recurrence, sepsis \\
\hline 12 & male & 86 & lung adenocarcinoma & left pneumonectomy & $6 \times 6$ & 33.6 & yes & disease recurrence, sepsis \\
\hline 13 & female & 69 & lung SQ cell cancer & right pneumonectomy & $6 \times 6$ & 15.2 & yes & disease recurrence, sepsis \\
\hline 14 & male & 91 & $\begin{array}{l}\text { tracheal laceration due to trau- } \\
\text { matic endotracheal intubation }\end{array}$ & no & $6 \times 6$ & 1.0 & yes & $\begin{array}{l}\text { mediastinitis } \\
\text { septic shock }\end{array}$ \\
\hline 15 & male & 62 & lung adenocarcinoma & left pneumonectomy & $6 \times 6$ & 5.0 & yes & septic shock \\
\hline 16 & male & 31 & Wegener's granulomatosis & right pneumonectomy & $6 \times 6$ & 0.1 & yes & septic shock \\
\hline 17 & male & 67 & lung adenocarcinoma & left pneumonectomy & $6 \times 6$ & 4.5 & yes & disease recurrence, sepsis \\
\hline
\end{tabular}

$\mathrm{SQ}=$ Squamous; $\mathrm{RML}=$ right middle lobe; $\mathrm{LUL}=$ left upper lobe; $\mathrm{LLL}=$ left lower lobe; $\mathrm{RUL}=$ right upper lobe; $\mathrm{LMSB}=$ left main stem bronchus; $\mathrm{CA}=$ carcinoma.

vice made of 144 nitinol mesh wires that allow the device to compress inside a catheter and, when released from the delivering catheter, return to its shape to occlude the target vessel or in our case the airway lumen [21-23]. The device has platinum markers on both ends. A stainless-steel micro screw is welded to one of the platinum marker bands, which allows attachment to the $135-\mathrm{cm}$ long delivery cable. The AVP is available in diameters ranging from 4 to $16 \mathrm{~mm}$ (in 2 - $\mathrm{mm}$ increments).

Technique of Deployment - AVP

The size and type of AVP chosen was according to the diameter of the bronchial stump and the length of the fistula with the intent that the device would completely cover the fistulous connection and fit inside the stump [17]. The delivery cable was introduced through the working channel of the bronchoscope allowing direct visualization of the deployment. Once positioned by holding the delivery shaft steady and pushing the plug forward, the AVP was released by rotating the delivery cable counterclockwise under both direct vision and fluoroscopic guidance. Following its deployment, a dynamic bronchogram was performed by injecting contrast medium through a catheter to check device position in relation to the stump and fistula.

The bronchoscope and sheath were removed from the airways, and the patients were transferred to the recovery room and discharged following a chest X-ray.

\section{Results}

The clinical and demographic characteristics of the study patients $(n=31)$ are provided in tables 1 and 2 . Of the 31 BPF patients, 19 were treated by $\mathrm{AD}$ and 
Table 2. Clinical and demographic characteristics of the 12 BPF patients with AVD placement

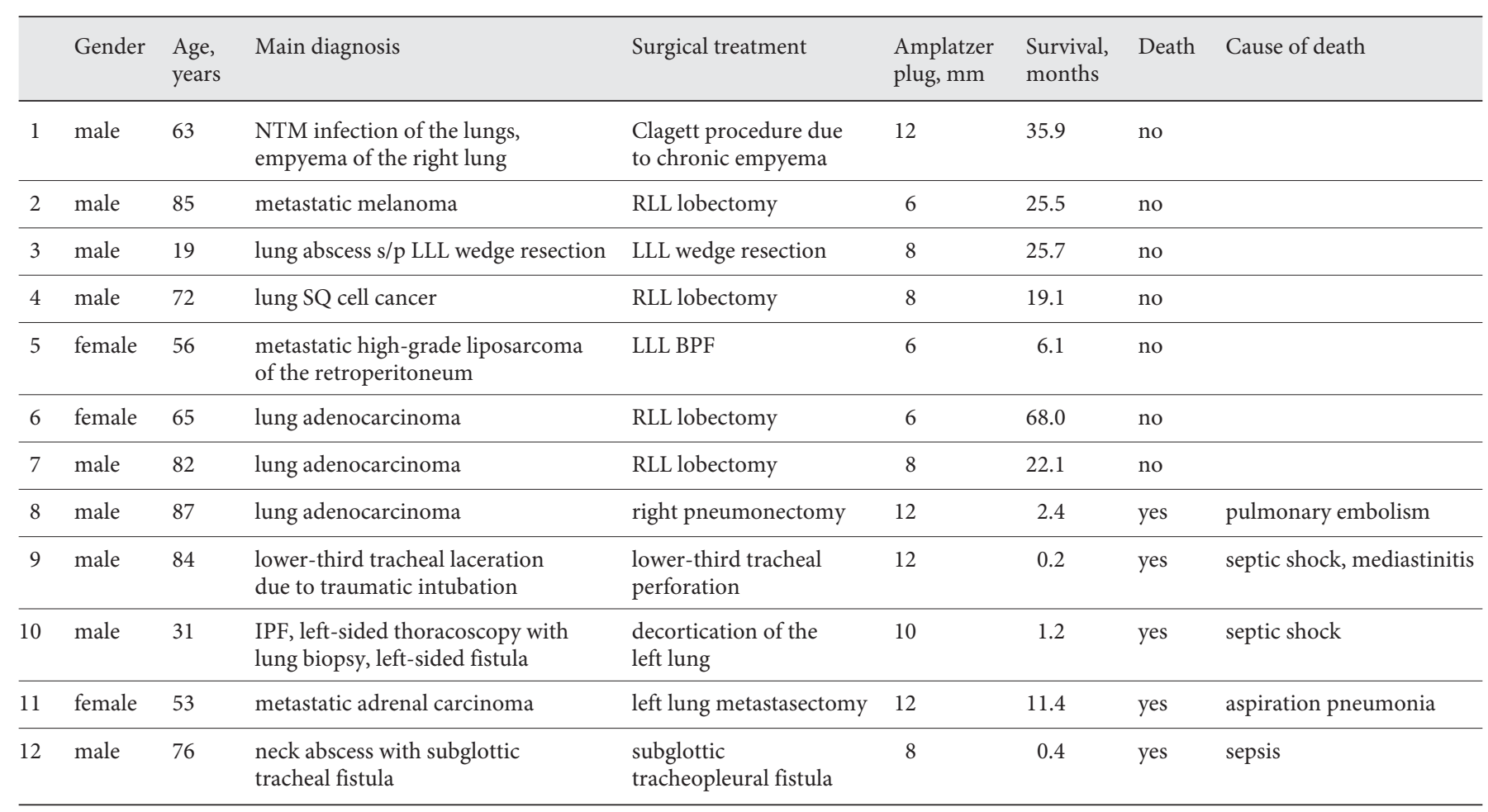

NTM = Nontuberculous mycobacteria; s/p = status post; SQ = squamous; RLL = right lower lobe; LLL = left lower lobe; IPF = idiopathic pulmonary fibrosis.

12 by AVP. The mean age of the entire cohort $( \pm S D)$ was $66.7 \pm 17.1$ years, with a notable male predominance (male/female ratio: $26: 5$ ). The vast majority of patients $(19=61 \%)$ developed BPF following lung resection for non-small lung cancer. There was an equal distribution of BPF occurrence following either right or left pneumonectomy (7 cases in each). The median time from surgery to development of postoperative BPF was 3 months (range 1-13 months). None of the patients has undergone a previous surgical attempt to close the BPF.

\section{$A D \operatorname{Group}(n=19)$}

The main etiology for the development of BPF in this subgroup of patients was lobectomy due to lung cancer $(\mathrm{n}=4)$, pneumonectomy for lung cancer $(\mathrm{n}=12)$, pneumonectomy due to necrotizing granulomatosis with polyangiitis (Wegener's granulomatosis; $\mathrm{n}=1$ ), tracheal laceration and tracheopleural fistula due to traumatic endotracheal intubation $(\mathrm{n}=1)$, and broncho-neoesophageal-pleural fistula due to left main stem bronchus invasion by esophageal cancer $(\mathrm{n}=1)$.

\section{AVP Group ( $n=12)$}

This subgroup of the study cohort included patients with BPF following lobectomy due to either primary lung cancer $(\mathrm{n}=4)$, right pneumonectomy due to non-small cell lung cancer $(\mathrm{n}=1)$, or lung resection due to secondary lung metastases $(n=2)$, wedge resection due to a lung abscess $(n=1)$, decortications of the lung due to empyema $(n=2)$, tracheal laceration due to traumatic intubation $(n=1)$ and metastatic high-grade liposarcoma of the retroperitoneum $(\mathrm{n}=1)$.

\section{Procedure Outcome}

In all but 1 patient, the bronchoscopic procedure was successful; the initial endoscopic result was satisfactory and symptoms related to BPF disappeared following closure by AD or AVP.

In 1 patient with a large stump-related BPF who developed BPF following right pneumonectomy due to granulomatosis with polyangiitis (Wegener's granulomatosis), an attempt was made to seal the fistula with a $6 \times 6 \mathrm{~mm}$ $\mathrm{AD}$. The procedure was unsuccessful and the patient died 3 days following deployment of the device from sepsis. 

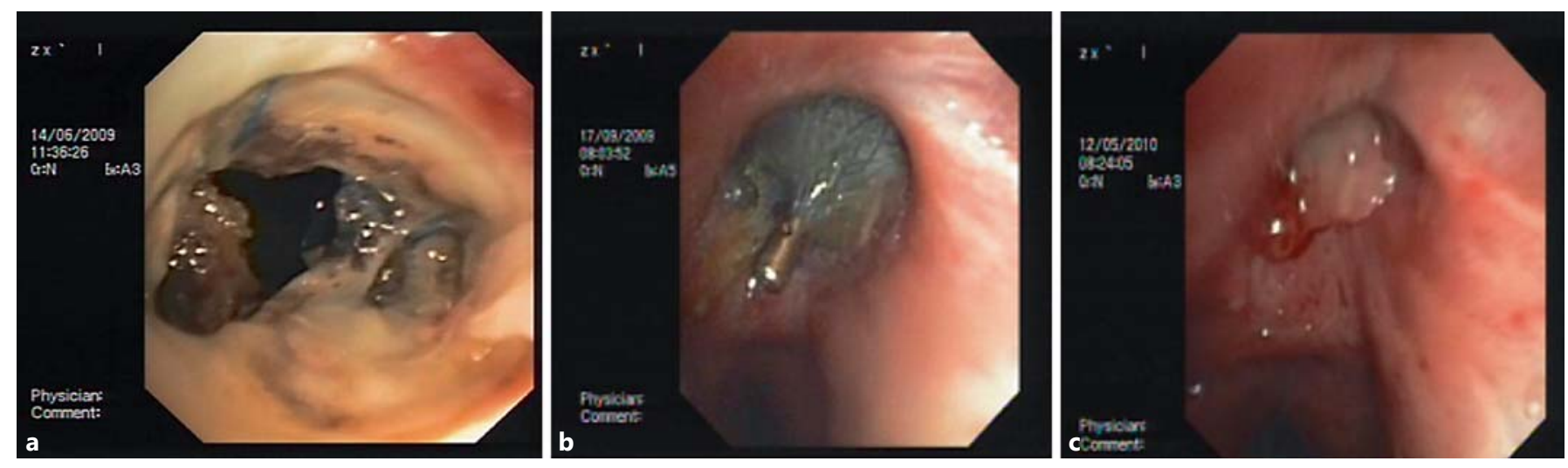

Fig. 1. A large BPF following right upper lobectomy before (a) and after $\mathrm{AD}$ placement (b); at the 8-month follow-up, it is completely covered with granulation tissue $(\mathbf{c})$.

Fig. 2. A small BPF following right pneumonectomy after AVP (a) and covered with granulation tissue at 12 months (b).
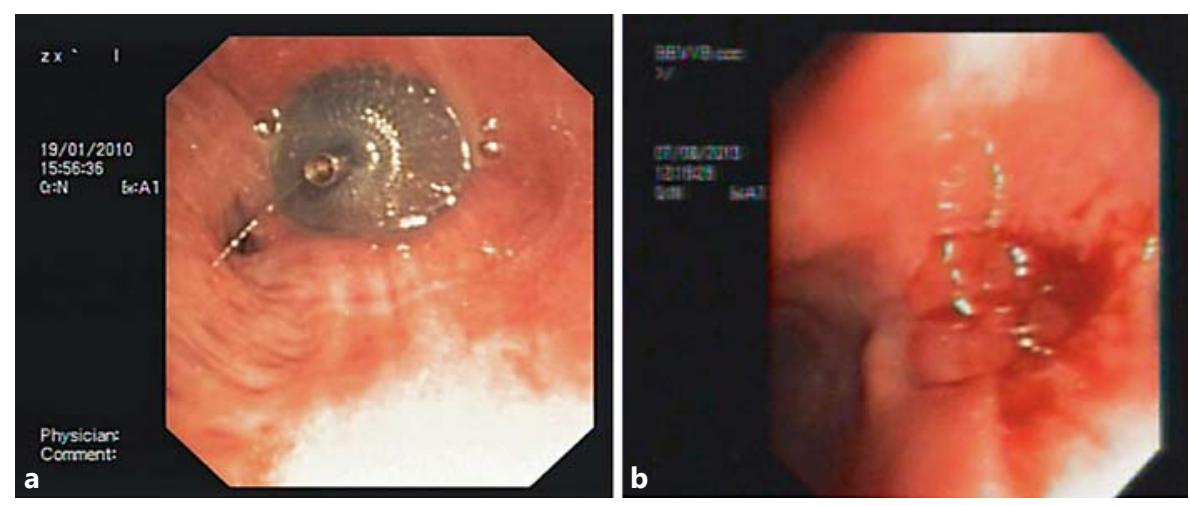

The immediate success rate was $96 \%$ and symptoms related to BPF disappeared in 30 of the 31 patients following closure by one of the devices. No immediate procedure-related mortality was observed; short-term $(<30$ days) mortality was $13 \%$ (4/31). Early mortality was due to multiorgan failure and sepsis in all cases.

The first follow-up surveillance bronchoscopy was performed 4 weeks following the procedure and every 3-6 months thereafter. In none of the cases the devices needed repositioning or removal, and device migration was not observed. Within 6 months postoperatively, all the devices were covered by granulation tissue. Representative figures of AD and AVP treatment immediately following deployment and at the 6-month surveillance bronchoscopy are presented in figures 1 and 2 .

The average follow-up period was 17.6 months (range 1-68 months). As of the writing of the paper, 14 patients (45\%) are still alive, 7 patients in each subgroup. Of 12 patients who had died $>30$ days after the procedure, the mortality was directly attributed to cancer recurrence in
5 patients (41\%), whereas mortality was mostly $(6 / 7,85 \%)$ due to sepsis in the remaining patients. No patient died due to BPF recurrence.

We did not notice any association between timing of the intervention in relation to BPF occurrence and success rate.

\section{Discussion}

In the current report, we explored the long-term outcome of a novel minimally invasive modality for treating $\mathrm{BPF}$ using $\mathrm{AD}$ and AVP. The technique described herein enables direct visualization of the fistula by flexible bronchoscopy and fluoroscopy simultaneously. The procedures were performed on an outpatient basis under moderate sedation and were well tolerated by the patients. The AD and AVP are designed to provide effective closure of the defect, and both occluders can be fully deployed, retrieved and repositioned prior to de- 
tachment, which is an important advantage for accurate positioning and coverage of the fistula. The procedures were carried out with very low immediate complication rate, and excellent short-term and long-term success rates.

Endoscopic treatment of BPF is based on the delivery of biological glue, coils, covered stents and sealants to the BPF site [5-14]. Other minimally invasive treatment options include the use of one-way endobronchial valves that are also used for the treatment of emphysema (Spiration and Zephyr) and endobronchial Watanabe spigots [12-14]. The degree of success is variable and depends on the underlying disease and the proximity and size of the fistulae, with larger fistulae having poor closure rates [7-11]. A large $\mathrm{BPF}$ is apparently particularly challenging. They are not amenable to closure with small devices such as coils or glue because of their poor stability in the lesion. The lack of a framework can cause the glue to spill over into the pleural space or into the contralateral bronchi. Coils are more suitable for small fistulae but do not anchor well in a large central lesion. Following our initial report using AD to seal BPF [15, 16], several other groups [24-26] have described their experience with this technique. Scordamaglio et al. [24] described a 53-year-old patient who underwent right upper lobectomy due to aspergilloma with hemoptysis and developed BPF at the right upper lobe stump. The patient was successfully treated by bronchoscopic closure of the fistula using an $\mathrm{AD}$ and was doing well at the 6-month follow-up. Gulkarov et al. [25] described a 68 -year-old patient with a right main stem BPF following pneumonectomy due to carcinoma. The authors described the successful closure of the BPF using minimally invasive bronchoscopic implantation of an $\mathrm{AD}$. Tedde et al. [26] reported their successful experience in closing a BPF following right upper lobectomy due to aspergilloma in a 53-year-old patient using an Occlutech Figulla ASD occlude, which is similar to the AD described.
The follow-up period for these patients was limited to a few months.

Previously, we have treated 5 patients with a small short-necked BPF using AVP closure [17]. The AVP is designed to provide effective closure of the defect, and, owing to the large range of device sizes, they can be appropriately matched to the diameter and length of the BPF. Following our experience, Spiliopoulos et al. [27], described successful sealing of large postoperative BPF in a 63-year-old man, but similar to our report, the followup period was limited.

Consequently, following our initial reports [15-17] and in view of the encouraging early results, a longer follow-up was warranted to ensure the durability of the procedure and to exclude potential long-term complications such as infections, device migration and occurrence of iatrogenic fistulae due to the device in neighboring vascular and mediastinal structures. In the current study on an extended average follow-up period of $>17$ months (with a maximal follow-up period of $>5$ years), we observed no such complications. Moreover, according to our experience, the device induces local granulation tissue formation that potentiates its occluding properties without compromising airway patency.

Based on the current data from a long-term clinical follow-up and surveillance bronchoscopic procedures, the technique described herein provides additional data regarding the safety and efficacy of this minimally invasive modality.

The ease of AD and AVP implantation under moderate sedation at the bronchoscopy suite adds this novel technique to the armamentarium of modalities for the minimally invasive treatment of BPF.

\section{Conflicts of Interest}

The authors have no conflicts of interest to disclose.

\section{References}

1 Cerfolio RJ: The incidence, etiology and prevention of postresectional bronchopleural fistula. Semin Thorac Cardiovasc Surg 2001;13: 3-7.

2 Pierson DJ, Horton CA, Bates PW: Persistent bronchopleural fistula air leak during mechanical ventilation: a review of 39 cases. Chest 1986;90:321-323.

-3 Sirbu H, Busch T, Aleksic I, Schreiner W, Oster O, Dalichau H: Bronchopleural fistula in the surgery of non-small cell lung cancer: in- cidence, risk factors and management. Ann Thorac Cardiovasc Surg 2001;7:330-336.

-4 Sonobe M, Nawkagawa M, Ichinose M, Ikegami N, Nagasawa M, Shindo T: Analysis of risk factors in bronchopleural fistula after pulmonary resection for primary lung cancer. Eur J Cardiothorac Surg 2000;18:519523.

5 Dutau H, Breen DP, Gomez C, Thomas PA, Vergnon JM: The integrated place of tracheobronchial stents in the multidisciplinary man- agement of large post-pneumonectomy fistulas: our experience using a novel customized conical self-expandable metallic stent. Eur J Cardiothorac Surg 2011;39:185-189.

6 Lois M, Noppen M: Bronchopleural fistula: an overview of the problem with special focus on endoscopic management. Chest 2005; 128 : 3955-3965.

7 McManigle JE, Fletcher GL, Tenholder MF: Bronchoscopy in the management of bronchopleural fistula. Chest 1990;97:1235-1238. 
8 Paul S, Talbot SG, Carty M, Orgill DP, Zellos L: Bronchopleural fistula repair during Clagett closure utilizing a collagen matrix plug. Ann Thorac Surg 2007;83:1519-1521.

-9 Tao H, Araki M, Sato T, Morino S, Kawanami R, Yoshitani M, Nakamura T: Bronchoscopic treatment of postpneumonectomy bronchopleural fistula with collagen screw plug. J Thorac Cardiovasc Surg 2006;132:99-104.

10 Ferguson JS, Sprenger K, Van Natta T: Closure of a bronchopleural fistula using bronchoscopic placement of an endobronchial valve designed for the treatment of emphysema. Chest 2006;129:479-481.

11 Ranu H, Gatheral T, Sheth A, Smith EE, Madden BP: Successful endobronchial seal of surgical bronchopleural fistulas using BioGlue. Ann Thorac Surg 2009;88:1691-1692.

-12 Bellato V, Ferraroli GM, Infante MV, Cariboni U, Spoto MR, Alloisio M, Bordone G: Management of postoperative bronchopleural fistula with a tracheobronchial stent in a patient requiring mechanical ventilation. Intensive Care Med 2010;36:721-722.

13 Chae EY, Shin JH, Song HY, Kim JH, Shim TS, Kim DK: Bronchopleural fistula treated with a silicone-covered bronchial occlusion stent. Ann Thorac Surg 2010;89:293-296.

14 Yoichi W, Keisuke M, Akihiko T, Reiko K, Shunkichi H: Bronchial occlusion with endobronchial Watanabe spigot. J Bronchol 2003; 10:264-267.
15 Kramer MR, Peled N, Shitrit D, Atar E, Saute M, Shlomi D, Amital A, Bruckheimer E: Use of Amplatzer device for endobronchial closure of bronchopleural fistulas. Chest 2008; 133:1481-1484.

16 Fruchter O, Kramer MR, Dagan T, Raviv Y, Abdel-Rahman N, Saute M, Bruckheimer E: Endobronchial closure of bronchopleural fistulae with Amplatzer devices: our experience and literature review. Chest 2011;139:682687.

17 Fruchter O, Bruckheimer E, Raviv Y, Rosengarten D, Saute M, Kramer MR: Endobronchial closure of bronchopleural fistulas with Amplatzer vascular plug. Eur J Cardiothorac Surg 2012;41:46-49.

18 Matitiau A, Birk E, Kachko L, et al: Transcatheter closure of secundum atrial septal defects with the Amplatzer septal occluder: early experience. Isr Med Assoc J 2001;3:32-35.

19 Butera G, Chessa M, Carminati M: Percutaneous closure of ventricular septal defects. Cardiol Young 2007;17:243-253.

20 Han YM, Gu X, Titus JL, et al: New self-expanding patent foramen ovale occlusion device. Catheter Cardiovasc Interv 1999;47: 370-376.
21 Hart JL, Aldin Z, Braude P, Shovlin CL, Jackson J: Embolization of pulmonary arteriovenous malformations using the Amplatzer vascular plug: successful treatment of 69 consecutive patients. Eur Radiol 2010;20:2663-2670.

22 Cil B, Peynirciolu B, Canyiit M, Geyik S, Ciftçi T: Peripheral vascular applications of the Amplatzer vascular plug. Diagn Interv Radiol 2008; 14:35-39.

23 Rimon U, Heldenberg E, Golan G, Shinfeld A, Garniek A: Amplatzer vascular plug: expanding the applications spectrum. Cardiovasc Intervent Radiol 2008;31:S84-S87.

24 Scordamaglio PR, Tedde ML, Minamoto H, Pedra CA, Jatene FB: Endoscopic treatment of tracheobronchial tree fistulas using atrial septal defect occluders: preliminary results. J Bras Pneumol 2009;35:1156-1160.

25 Gulkarov I, Paul S, Altorki NK, Lee PC: Use of Amplatzer device for endobronchial closure of bronchopleural fistulas. Interact Cardiovasc Thorac Surg 2009;9:901-902.

26 Tedde ML, Scordamaglio PR, Minamoto H, et al: Endobronchial closure of total bronchopleural fistula with Occlutech Figulla ASD N device. Ann Thorac Surg 2009;88:e25-e26.

27 Spiliopoulos S, Krokidis M, Gkoutzios P, McGrath A, Ahmed I, Karunanithy N, Routledge T, Sabharwal T, Adam A: Successful exclusion of a large bronchopleural fistula using an Amplatzer II vascular plug and glue embolization. Acta Radiol 2012;53:406-409. 